\title{
Spo13 regulates cohesin cleavage
}

\author{
Brian H. Lee, Angelika Amon, ${ }^{2}$ and Susanne Prinz ${ }^{1}$ \\ Center for Cancer Research, Howard Hughes Medical Institute, Massachusetts Institute of Technology, \\ Cambridge, Massachusetts 02139, USA
}

\begin{abstract}
A key aspect of meiotic chromosome segregation is that cohesin, the protein complex that holds sister chromatids together, dissociates from chromosome arms during meiosis $I$ and from centromeric regions during meiosis II. The budding yeast protein Spo13 plays a key role in preventing centromeric cohesin from being lost during meiosis I. We have determined the molecular basis for the metaphase arrest obtained when SPO13 is overexpressed during the mitotic cell cycle. Overexpression of SPO13 inhibits anaphase onset by at least two mechanisms. First, Spo13 causes a transient delay in degradation of the anaphase inhibitor Pds1. Second, Spo13 inhibits cleavage of the cohesin subunit Scc1/Med1 or its meiosis-specific homolog, Rec8, by the separase Esp1. The finding that Spo13 did not prevent cleavage of another Esp1 substrate, Slk19, suggests that overexpression of $S P O 13$ is sufficient to prevent cohesin cleavage by protecting specific substrates from separase activity.
\end{abstract}

[Key Words: Spo13; separin; cohesin; sister-chromatid; segregation; meiosis; S. cerevisiae]

Received March 5, 2002; revised version accepted May 13, 2002.

To generate two daughter cells with the exact same complement of chromosomes, it is critical that sister chromatids remain tightly associated prior to chromosome segregation and promptly dissociate as nuclear division commences during anaphase. Essential to this process is the multisubunit protein complex known as cohesin, which provides the physical linkage between sister chromatids. In budding yeast, the cohesin complex consists of at least four subunits, Smc1, Smc3, Scc1/ Mcd1, and Scc3 (for review, see Uhlmann 2001).

Cohesion between sister chromatids is established during DNA replication (Uhlmann and Nasmyth 1998) and is maintained until the onset of anaphase, when chromosome segregation is initiated by the loss of cohesion. At the onset of anaphase, the cohesin subunit Scc1/ Mcd1 is cleaved by a protease known as separase (Esp1 in budding yeast), allowing sister chromatids to separate (for review, see Nasmyth et al. 2000). The activity of Esp1 is controlled by the anaphase inhibitor securin (Pds1 in budding yeast), which binds to and inhibits Esp1. A ubiquitin ligase called the Anaphase Promoting Complex or Cyclosome (APC/C), in association with the WD40 repeat protein Cdc20, targets Pds1 for degradation at the metaphase-anaphase transition (for review, see Peters 1998; Cohen-Fix 2001), thereby freeing Esp1 to initiate chromosome segregation and mitotic exit.

In contrast to mitosis, where cohesion is lost along the

\footnotetext{
${ }^{1}$ Present address: Institute for Systems Biology, 1441 North 34th Street, Seattle, WA 98103, USA.

${ }^{2}$ Corresponding author.

E-MAIL angelika@mit.edu; FAX (617) 258-6558.

Article and publication are at http://www.genesdev.org/cgi/doi/10.1101/ gad.989302.
}

entire length of the chromosome by the onset of anaphase, sister-chromatid cohesion is lost in a stepwise manner during meiosis (for review, see Miyazaki and Orr-Weaver 1994; Lee and Orr-Weaver 2001). Sister-chromatid cohesion is lost along chromosome arms at the onset of anaphase I, but centromeric cohesion is retained until the onset of anaphase II. Loss of cohesion on chromosome arms is required for homologs to segregate from each other (Buonomo et al. 2000), whereas retention of centromeric cohesion between sister chromatids beyond anaphase I and its subsequent loss at the onset of anaphase II are critical for proper segregation of sister chromatids during meiosis II (Tang et al. 1998). Budding yeast cells accomplish this stepwise loss of cohesion during meiosis in part by replacing the mitotic cohesin subunit Scc1/Mcd1 with a meiosis-specific homolog, Rec8. When REC8 is replaced by SCC1/MCD1, sister-chromatid cohesion is lost along the entire length of the chromosome during the first meiotic division, suggesting that retention of cohesion around centromeres is a specific feature of Rec8-containing cohesin complexes (Toth et al. 2000). As cleavage of Scc1/Mcd1 is required for chromosome segregation in mitosis, Rec8 cleavage by Espl is required for chromosome segregation during meiosis (Buonomo et al. 2000). Cleavage of Rec8 at chromosome arms is necessary for anaphase I to occur, and cleavage of Rec8 at centromeric regions is also likely to be required for sister chromatids to separate during meiosis II. The mechanisms that bring about this differential control of Rec8 cleavage are unknown.

SPO13 is required to inhibit sister-chromatid separation during meiosis I. Cells lacking SPO13 undergo a single meiotic division with a mixed chromosome segregation pattern; some chromosomes segregate preferen- 
tially in an equational manner (sister chromatids are separated), whereas others preferentially segregate reductionally (homologs are separated; Klapholz and Esposito 1980a,b; Hugerat and Simchen 1993). Furthermore, in spo13s mutants, Rec8 is lost at the chromosome arms and centromeres simultaneously (Klein et al. 1999).

SPO13 overexpression during the mitotic and meiotic cell cycle leads to a metaphase arrest and metaphase I delay, respectively (McCarroll and Esposito 1994). To examine how Spol3 functions to regulate chromosome segregation, we analyzed the consequences of SPO13 overexpression on mitotic cell cycle progression. We find that when overexpressed during mitosis, SPO13 inhibits anaphase onset by at least two mechanisms. High levels of Spo13 cause a transient delay in the degradation of the anaphase inhibitor Pds1. Importantly, Spo13 also inhibits the cleavage of the cohesin subunits Scc1/Mcd1 and Rec8 in a PDS1-independent manner but does not prevent cleavage of another Esp1 substrate, Slk19. Our results indicate that overexpression of SPO13 is sufficient to specifically inhibit cleavage of the cohesin subunits Scc1/Mcd1 and Rec8 by Esp1.

\section{Results}

Overexpression of SPO13 arrests cells in metaphase

To determine the molecular basis whereby high levels of Spo13 prevent cell cycle progression, we analyzed the consequences of expressing SPO13 from the galactoseinducible GAL1-10 promoter (GAL-SPO13) or the tetracycline/doxycycline-repressible TET promoter system (TET-SPO13). Cells were arrested in the $\mathrm{G}_{1}$ stage of the cell cycle with $\alpha$-factor pheromone and released into the cell cycle under conditions where SPO13 was expressed to high levels. Progression through $S$ phase was not affected as judged by FACS analysis (data not shown), but progression through mitosis was inhibited (Fig. 1A,C). Cells overexpressing SPO13 arrested as large budded cells with metaphase spindles and an undivided nucleus (Fig. 1A,C), indicating that high levels of Spo13 block entry into anaphase.

Anaphase onset is triggered by the degradation of securin Pds1, which liberates Esp1 to initiate sister-chromatid separation (for review, see Peters 1998). To determine if the SPO13-induced metaphase arrest was caused by Pds1 stabilization, we analyzed Pds1 protein levels in TET-SPO13 cells. Pds1 protein levels initially remained elevated during the arrest but then declined despite cells remaining arrested in metaphase (Fig. 1B,C). Levels of the mitotic cyclin $\mathrm{Clb} 2$ remain high during the SPO13induced arrest, and the cyclin-dependent kinase (CDK) inhibitor Sicl did not accumulate, indicating that exit from mitosis did not occur (Fig. 1B). These results suggest that elevated Pds1 levels are in part responsible for the metaphase arrest observed in cells overexpressing SPO13. The finding that Pds1 protein levels declined and cells were nevertheless arrested with metaphase-like spindles raised the possibility that high levels of Spo13 also prevent anaphase onset by means other than inhibiting Pds1 degradation.
Pds1 stabilization caused by high levels of Spo13 is largely independent of the DNA damage and mitotic spindle checkpoints

DNA or mitotic spindle damage lead to the activation of the DNA damage and spindle checkpoints, respectively, both of which bring about cell cycle arrest through inhibition of Pds1 degradation (Gardner and Burke 2000; for review, see Melo and Toczyski 2002). To determine whether the stabilization of Pds1 observed in cells overexpressing SPO13 was caused by activation of either checkpoint, we analyzed Pds1 levels in TET-SPO13 cells deleted for the DNA damage checkpoint component $R A D$ 9 or the mitotic spindle checkpoint component $M A D 1$. Pds1 levels remained elevated in cells lacking $R A D 9$ (data not shown). Inactivation of the mitotic spindle checkpoint, or both the DNA damage and spindle checkpoints, caused a minor decline in Pds 1 protein levels in cells overexpressing SPO13 (Fig. 1B; data not shown). We conclude that the stabilization of Pds1 brought about by high levels of Spol3 is not caused by activation of the DNA damage checkpoint and is only in part caused by activation of the mitotic spindle checkpoint. Inactivation of either checkpoint or both did not alleviate the metaphase arrest caused by overexpression of SPO13 (Fig. 1C; data not shown) indicating that mechanisms other than activation of these checkpoints prevented entry into anaphase in cells expressing high levels of Spo13.

\section{High levels of SPO13 inhibit anaphase entry in the absence of PDS1}

The observation that cells overexpressing SPO13 arrest in metaphase despite declining levels of Pds1 raised the possibility that SPO13 could arrest cells in this stage of the cell cycle without stabilizing Pds1. To test this possibility directly, we examined the consequences of overexpressing SPO13 in cells lacking PDS1. GAL-SPO13 or TET-SPO13 cells lacking PDS1 arrested in metaphase as judged by mitotic spindle morphology (Fig. 1D; see Figs. 3C, 4A, below), showing that high levels of SPO13 can cause a metaphase arrest in the absence of PDS1.

\section{Overexpression of SPO13 prevents sister-chromatid separation by inhibiting Scc1/Mcd1 cleavage}

High levels of Spo13 could inhibit entry into anaphase by either preventing sister-chromatid separation or interfering with mitotic spindle elongation. To distinguish between these possibilities, we analyzed whether overexpression of SPO13 could arrest cells in metaphase in the absence of sister-chromatid cohesion. To inactivate cohesion between sister chromatids we used a temperature-sensitive allele of the SCC1/MCD1 cohesin subunit, mcd1-1 (Guacci et al. 1997). Loss of cohesion brought about by the mcd1-1 allele causes activation of the mitotic spindle checkpoint (Severin et al. 2001). Therefore, we analyzed the effects of the mcd1-1 mutation on the GAL-SPO13-induced metaphase arrest in 
Lee et al.

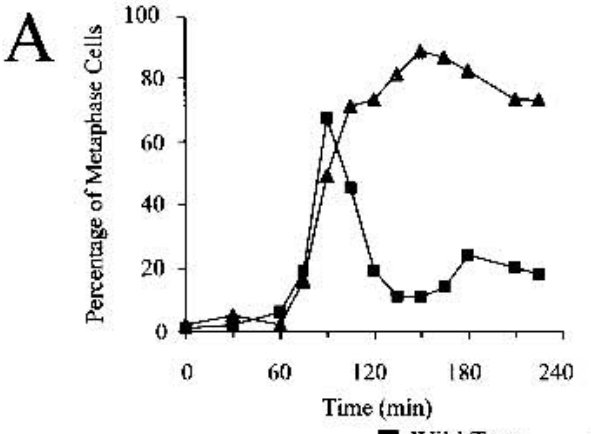

— Wild Type

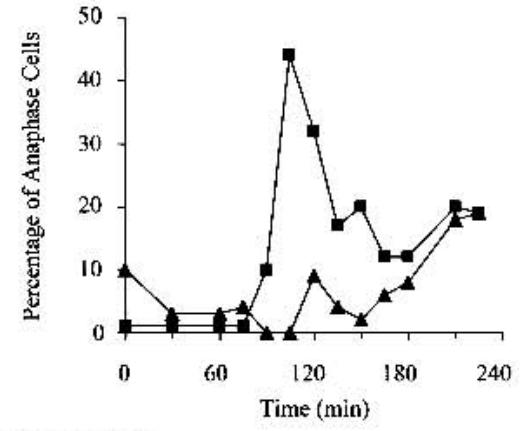

A GAL-SPOI3

B

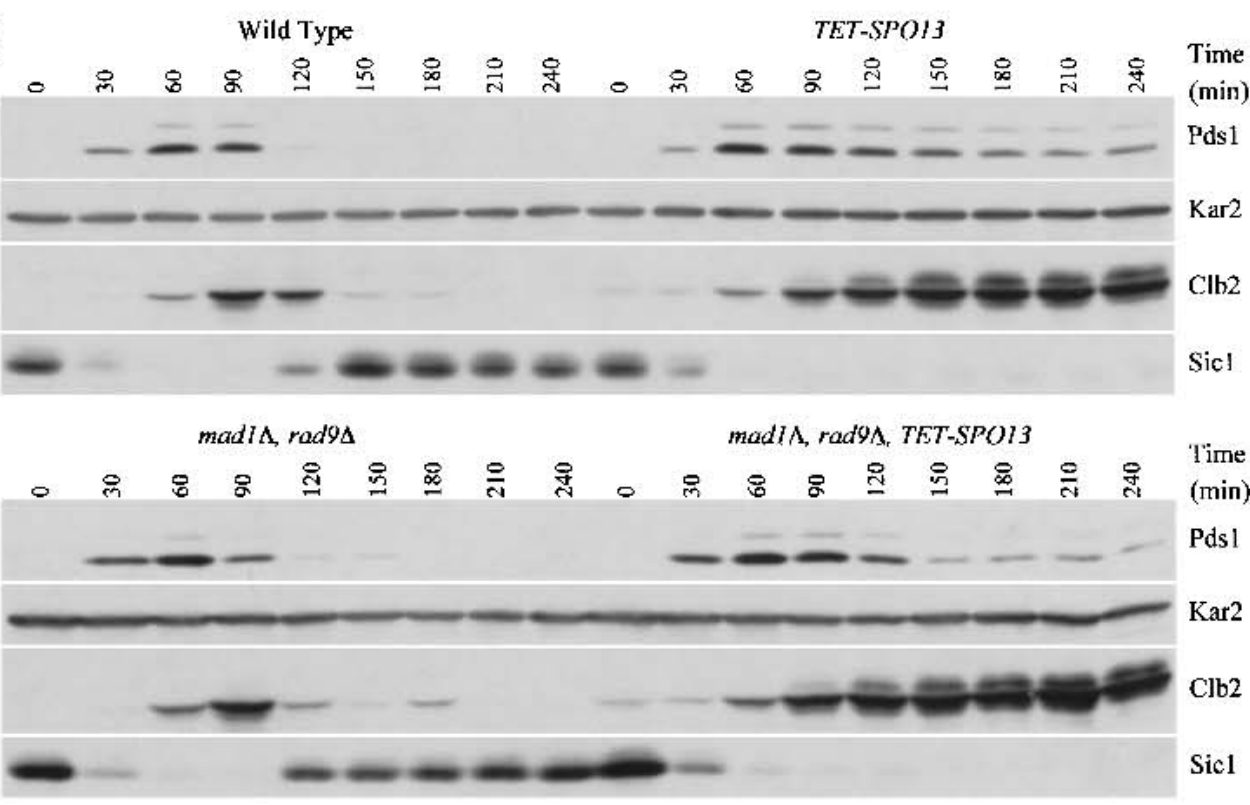

$\mathrm{C}$
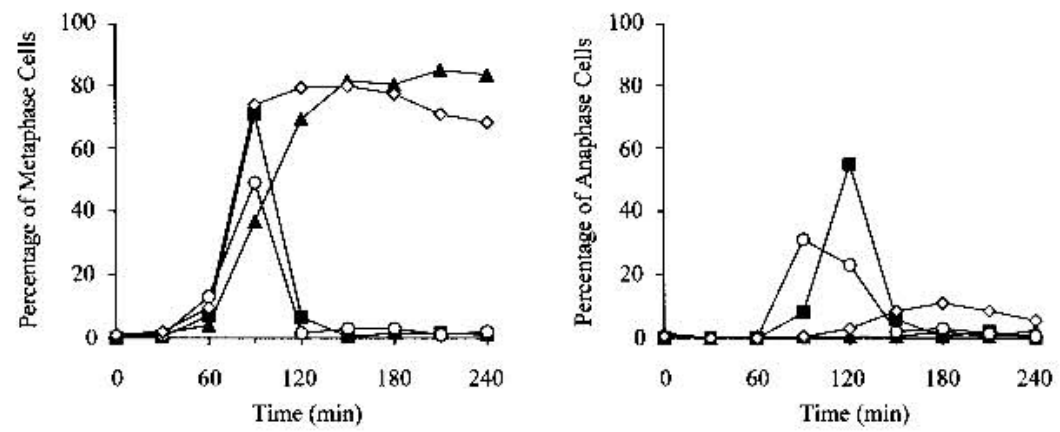

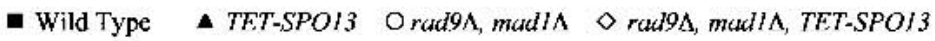

D

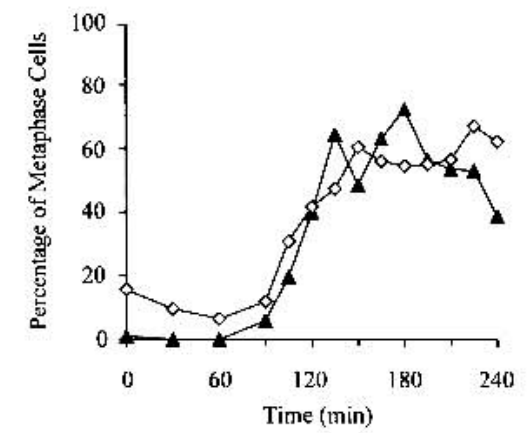

A GAL-SPOI3

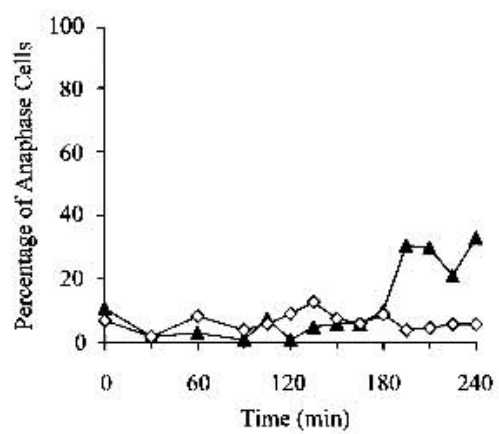

$\diamond p d s 1 \triangle, G A L-S P O I 3$

(Figure 1 legend on facing page) 
cells deleted for the mitotic spindle checkpoint component MAD1. Loss of sister-chromatid cohesion through heat inactivation of the mcd1-1 allele allowed GALSPO13 cells to enter anaphase (Fig. 2A). Cells were then delayed in exit from mitosis, as judged by the persistence of cells with anaphase spindles (Fig. 2A), which is consistent with the finding that cells expressing high levels of SPO13 accumulate high levels of the mitotic cyclin Clb2 and fail to accumulate the CDK inhibitor Sic1. Our results indicate that overexpression of SPO13 does not inhibit mitotic spindle elongation, but rather interferes with the dissolution of sister-chromatid cohesion and mitotic CDK inactivation.

At the onset of anaphase, the cohesin subunit Scc1/ Mcd1 is cleaved, and the cleavage products transiently accumulate during anaphase (Uhlmann et al. 1999). To determine whether overexpression of SPO13 inhibited the onset of anaphase by preventing Scc1/Mcdl cleavage, we analyzed whether Scc1/Mcd1 was cleaved in $G A L-$ SPO13 and TET-SPO13 cells. Upon release from a pheromone-induced $G_{1}$ arrest, the Scc1/Mcdl cleavage product was transiently observed in wild-type cells at the onset of anaphase, and simultaneously, full-length Scc1/ Mcdl levels declined (Fig. 2B; data not shown). In contrast, no Scc1/Mcd1 cleavage product was observed in cells overexpressing SPO13, and full-length Scc1/Mcd1 persisted (Fig. 2B; data not shown). The bulk of Scc1/ Mcd1 was also not cleaved in TET-SPO13 cells lacking PDS1 (see Fig. 4B below), showing that overexpression of SPO13 prevents Scc1/Mcdl cleavage and that it does so in part by means other than Pds1 stabilization.

To test directly whether the metaphase arrest caused by high levels of Spo13 was caused by inhibition of Scc1/ Mcdl cleavage, we analyzed whether cleavage of Sccl/ Mcdl was sufficient to induce anaphase entry in cells overexpressing SPO13. We used a SCC1/MCD1 allele in which one of the Esp1 cleavage sites is replaced by a cleavage site for the tobacco etch virus (TEV) protease (scc1-TEV268; Uhlmann et al. 2000). Cells overexpressing SPO13 and carrying a scc1-TEV268 construct as the sole source of $S C C 1 / M C D 1$ were released from a $G_{1}$ block, and production of the TEV protease (under the control of the GAL1-10 promoter) was induced when cells entered the TET-SPO13-induced metaphase arrest (110 min after release from $\alpha$-factor). Scc1/Mcdl cleavage occurred efficiently soon after induction of the TEV protease, as judged by the appearance of the Scc1/Mcd1 cleavage product, chromosome segregation, and mitotic spindle elongation (Fig. 2C, +Galactose). Exit from mitosis, however, did not occur, as Clb2 protein levels remained elevated and mitotic spindles did not disassemble (Fig. 2C), indicating that Spo13 overexpression also prevented mitotic CDK inactivation. Under conditions wherein expression of the TEV protease was inhibited (+Glucose), Scc1/Mcd1 cleavage did not occur and cells remained arrested in metaphase (Fig. 2C). Our results show that cleavage of Scc1/Mcd1 was sufficient to induce anaphase in TET-SPO13-arrested cells. We conclude that Spo13 inhibits anaphase by inhibiting Scc1/ Mcd1 cleavage. This inhibition is in part caused by a transient stabilization of Pds1 but is also caused by a PDS1-independent mechanism that affects cleavage of Scc1/Mcdl directly.

\section{Overexpression of SPO13 inhibits Rec8 cleavage}

During meiosis, Pds1 is degraded at the metaphase I-anaphase I transition, which liberates Esp1 to cleave the meiotic cohesin subunit Rec8 on chromosome arms (Buonomo et al. 2000; Salah and Nasmyth 2000). Thus, Pds1 is not likely to be involved in preventing Esp1 from cleaving Rec8 at centromeric regions during the first meiotic division. Spo13, on the other hand, is required for retention of centromeric cohesion during anaphase I (Klein et al. 1999). Our analysis of the effects of SPO13 overexpression on mitotic cell cycle progression showed that Spo13 inhibits cleavage of Scc1/Mcd1 in part through a PDS1-independent mechanism. The PDS1-independent mechanism whereby Spo13 inhibits Scc1/ Mcd1 cleavage during mitosis may be similar to the mechanism whereby Spo13 protects centromeric Rec8 during anaphase I. We were, unfortunately, not able to test the possibility that overexpression of SPO13 prevented Rec8 cleavage during the meiotic cell cycle directly. For reasons that are unclear, overexpression of SPO13 during meiosis resulted in a failure of cells to enter metaphase I, with the majority failing to initiate premeiotic S phase (B. Lee, unpubl.). This early meiotic cell cycle arrest made it impossible to test whether high levels of Spo13 interfered with Rec8 cleavage and anaphase I onset. Furthermore, deletion of PDS1 in meiosis caused cells to arrest in prophase I (data not shown).

To determine whether Spo13 could at least in principle inhibit Rec8 cleavage in a PDS1-independent manner, we expressed $R E C 8$ in vegetatively growing $p d s 1 \Delta$ cells from the $S C C 1 / M C D 1$ promoter and analyzed the

\footnotetext{
Figure 1. Overexpression of SPO13 causes cells to arrest in metaphase. $(A)$ Wild-type $(\mathbf{\square}, \mathrm{A} 940)$ and $G A L-S P O 13(\mathbf{\Lambda}$, A1813) cells were arrested in $\mathrm{G}_{1}$ with $\alpha$-factor $(5 \mu \mathrm{g} / \mathrm{mL})$ in YEPR (YEP $+2 \%$ raffinose). After $1 \mathrm{~h}$, galactose was added to $2 \%$ to induce $S P O 13$ expression. Cells were then released into medium containing galactose but lacking pheromone. The percentage of cells with metaphase (left graph)

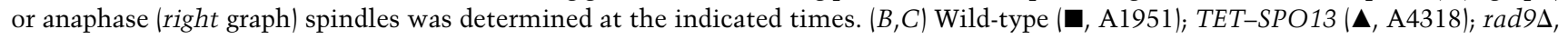
$\operatorname{mad} 1 \Delta(\mathrm{O}, \mathrm{A} 5309)$; and $\mathrm{rad} 9 \Delta, \operatorname{mad1\Delta }, \mathrm{TET}-S P O 13(\diamond, \mathrm{A} 5310)$ cells containing a PDS1-HA fusion were grown in YEPR containing doxycycline $(5 \mu \mathrm{g} / \mathrm{mL})$ to inhibit SPO13 expression. Cells were then washed to remove the doxycycline and arrested in $\mathrm{G}_{1}$ with $\alpha$-factor $(5 \mu \mathrm{g} / \mathrm{mL})$ for $4 \mathrm{~h}$, followed by release into YEPR lacking pheromone and doxycycline. After $100 \mathrm{~min}$, when cells had budded, $5 \mu \mathrm{g} / \mathrm{mL}$ $\alpha$-factor was added to prevent entry into the second cell cycle. The total amount of Pds1, Clb2, and Sic1 $(B)$, as well as the percentage of cells with metaphase $(C$, left graph) or anaphase $(C$, right graph) spindles were determined at the indicated times. Kar2 was used as a loading control in Western blot analyses. $(D) G A L-S P O 13(\boldsymbol{\Lambda}$, A1767) and GAL-SPO13 pds1s ( $\diamond$, A1801) cells were grown as described in $A$, and the percentage of cells with metaphase (left graph) or anaphase (right graph) spindles was determined.
} 
Lee et al.

A

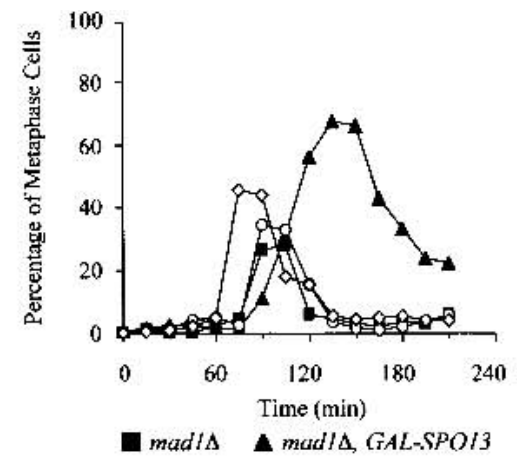

B

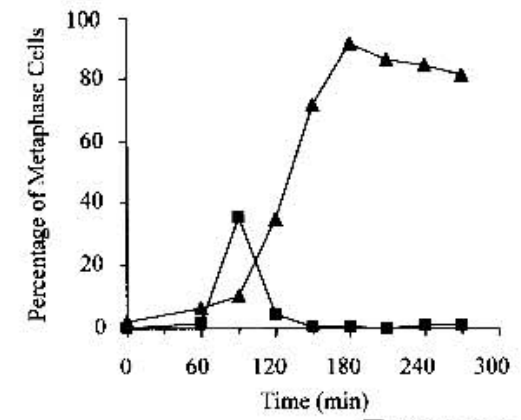

- Wild Type
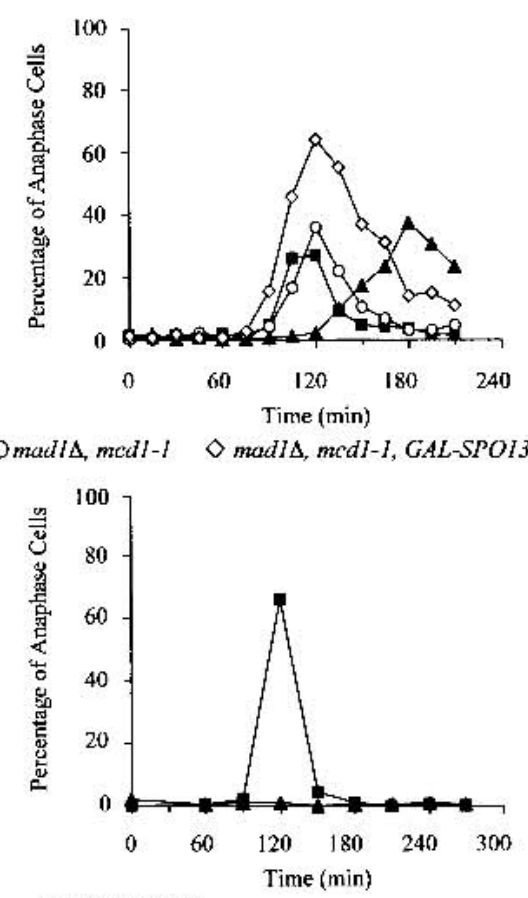

A TET-SPOI3

Wild Type

TET-SPO13

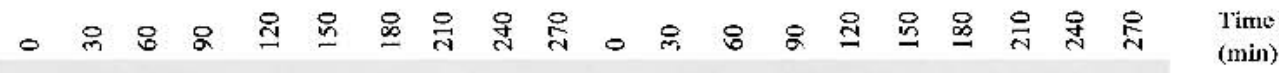

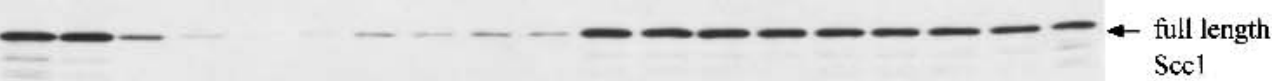

$\mathrm{Scc} 1$

$\operatorname{Cdc} 28$

C
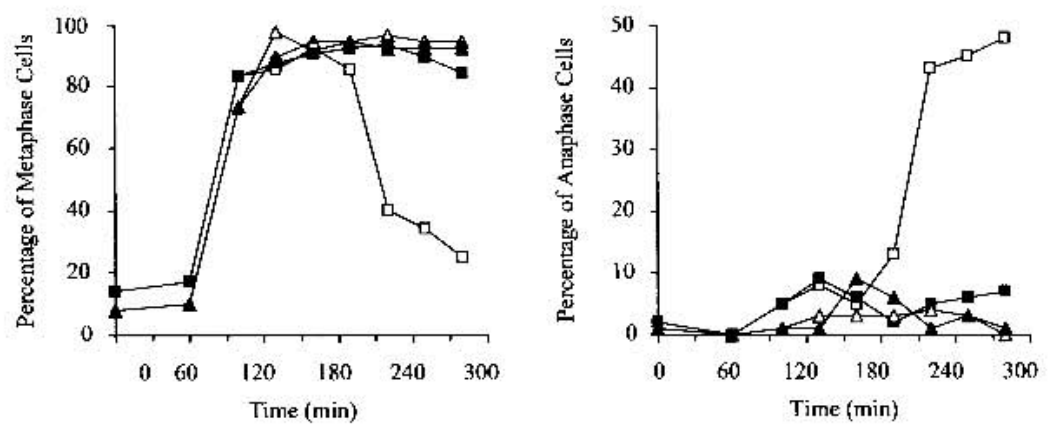

$\triangle T E T-S P O I 3$ Glu $\triangle T E T$-SPOI3 Gal $\square T E T-S P O 13$, GAL-TEV Glu QTET-SPOI3, GAL-TEV Gal

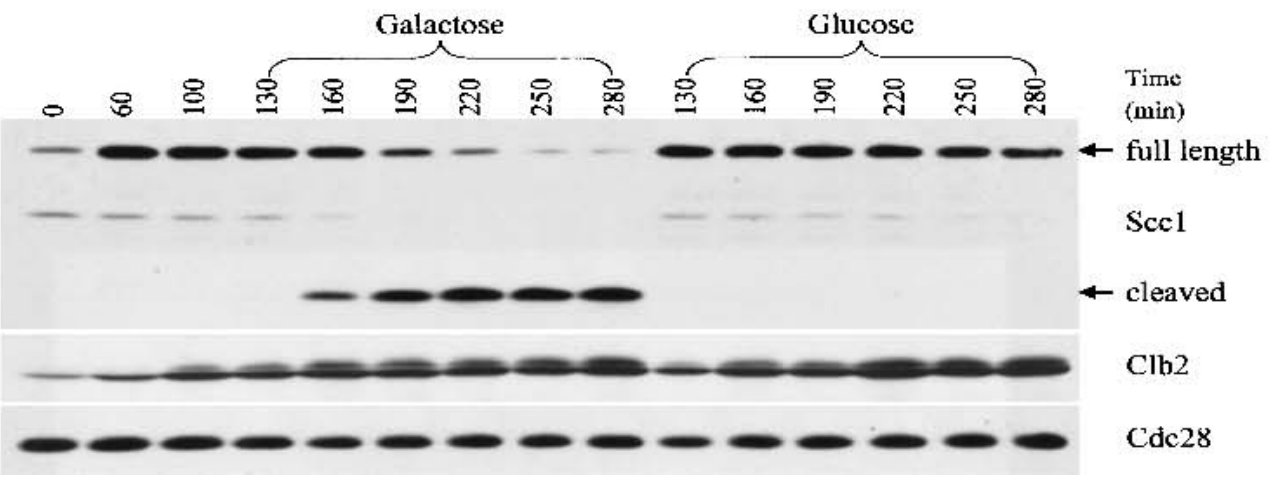

(Figure 2 legend on facing page) 
effects of SPO13 overexpression on Rec8 cleavage. We first determined whether Rec 8 is indeed cleaved by separase when expressed during mitosis. Upon release from a pheromone block, about half of the total amount of Rec8 protein was degraded during $S$ phase in a separase independent-manner (Fig. 3B, +Nocodazole). The remaining Rec 8 was degraded at the onset of anaphase in a separasedependent manner. Full-length Rec8 levels declined, and the Rec8 C-terminal cleavage product, which is the same as the C-terminal cleavage observed in cells undergoing meiosis (data not shown), appeared during anaphase onset. In contrast, in cells treated with nocodazole, which inhibits Esp1 activity (Uhlmann et al. 1999), or in cells carrying a temperature-sensitive esp1-1 mutant, fulllength Rec8 persisted, and the accumulation of the Rec8 C-terminal cleavage product was prevented (Fig. 3B; data not shown). In cells overexpressing SPO13, Rec8 behaved in a manner similar to that of cells treated with nocodazole (Fig. 3D). About half of the total amount of Rec8 was degraded during $S$ phase, but the remaining pool of Rec8 was stable (Fig. 3D). Furthermore, the Cterminal cleavage product of Rec8 was not observed, suggesting that high levels of Spo13 can prevent cleavage of the meiosis-specific cohesin subunit Rec8. This observation is also in agreement with the finding that overexpression of SPO13 prevented Rec8 from dissociating from chromosomes during mitosis (M. Shonn and A. Murray, pers. comm.; see accompanying paper by Shonn et al. in this issue).

\section{SPO13 overexpression does not prevent cleavage} of S1k19

The recent identification of another substrate of Esp1, the kinetochore protein Slk19 (Sullivan et al. 2001), enabled us to test whether Spo13 specifically inhibited cleavage of the cohesin subunits Scc1/Mcd1 and Rec8 or whether it inhibited Esp1 activity toward all its known substrates. We analyzed Spo13's effect on cleavage of Slk19 in cells lacking PDS1 to characterize specifically the consequences of SPO13 overexpression that occur independently of Pds1 stabilization. In contrast to Scc1/ Mcd1, Slk19 cleavage occurred in TET-SPO13 cells (Fig. 4B). As observed in earlier experiments, Spo13 also inhibited mitotic CDK inactivation, as Clb2-associated ki- nase activity remained elevated throughout the course of the experiment (Fig. 4B). We conclude that Spo13 specifically protects Rec8 and Scc1/Mcd1 from separase activity.

\section{Discussion}

Our results show that overexpression of SPO13 during mitosis inhibits sister-chromatid separation. SPO13 causes this metaphase arrest by two means. First, high levels of Spo13 cause a transient delay in Pds1 degradation. How Spo13 brings about the transient stabilization of Pds1 is not known. It is only to some extent through activation of the mitotic spindle checkpoint, as Pds1 protein levels persist for a slightly shorter time in TETSPO13 cells lacking the mitotic spindle checkpoint component MAD1. Second, SPO13 also prevented sisterchromatid separation in the absence of PDS1. We could exclude the possibility that this inability to enter anaphase was caused by defects in mitotic spindle elongation and showed that it was caused by a failure to cleave the cohesin subunit Scc1/Mcd1.

A key question is whether the mechanism in which high levels of Spo13 inhibit mitotic cell cycle progression provides insight into the function of Spo13 during meiosis. The finding that overexpression of SPO13 is sufficient to prevent cohesin cleavage suggests that Spo13 is a regulator of Rec8 cleavage during meiosis. However, overexpression of SPO13 alone does not recapitulate all aspects of cohesion regulation that occur during meiosis, as high levels of Spo13 prevent Scc1/Mcd1 cleavage during mitosis, but wild-type levels of Spo13 cannot protect Scc1/Mcd1 from being cleaved when SCC1/MCD1 is expressed instead of REC8 during meiosis (Toth et al. 2000). Furthermore, high levels of Spo13 appeared to prevent a large fraction of Sccl/Mcd1 and Rec8 from being cleaved rather than only the presumably small pool that localizes to centromeres (Figs. 3, 4). Clearly, therefore, overexpression of SPO13 during mitosis alone cannot bring about a meiosis-specific chromosome segregation pattern. However, our results nevertheless show that overexpression of SPO13 is sufficient to prevent Esp1 from cleaving Scc1/Mcd1 or Rec8. Importantly, the inhibition of cohesin cleavage brought about by Spo13 is, as in meiosis, independent of PDS1

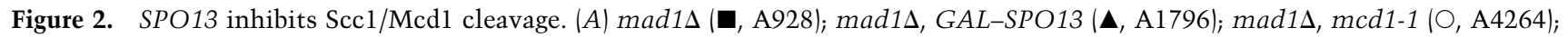
and mad1s, GAL-SPO13, mcd1-1 ( $\diamond, A 5413)$ cells were grown as described in Figure 1A except cells were released into YEPR medium containing galactose at $37^{\circ} \mathrm{C}$. The percentage of cells with metaphase (left graph) or anaphase (right graph) spindles was determined at

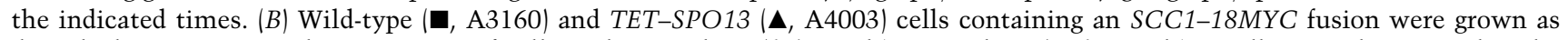
described in Figure 1B. The percentage of cells with metaphase (left graph) or anaphase (right graph) spindles was determined at the indicated times. The total amount of Scc1-Myc was determined by Western blot analysis. Cdc28 was used as a loading control. $(C)$

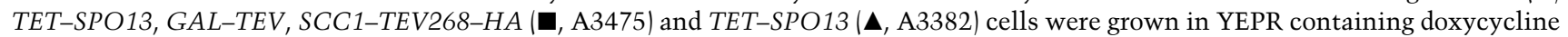
$(5 \mu \mathrm{g} / \mathrm{mL})$. Cells were then washed and grown for $2 \mathrm{~h}$ in YEPR lacking doxycycline to induce SPO13 expression. Then $5 \mu \mathrm{g} / \mathrm{mL} \alpha$-factor was added to arrest cells in $G_{1}$, followed by release into YEPR lacking pheromone and doxycycline. After 110 min, when cells were arrested in metaphase, the cultures were split and galactose was added to $2 \%$ (open symbols) or glucose to $2 \%$ (closed symbols) to induce or inhibit the expression of the TEV protease, respectively. The percentage of cells with metaphase (left graph) and anaphase spindles (right graph) was determined at the indicated times. The total amount of Scc1-HA and Clb2 for strain A3475 was determined by Western blot analysis. Cde28 was used as a loading control.
} 
Lee et al.

A

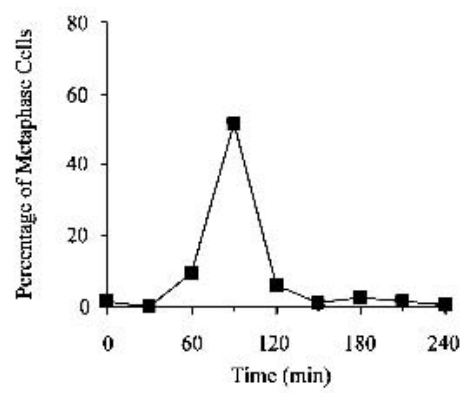

B

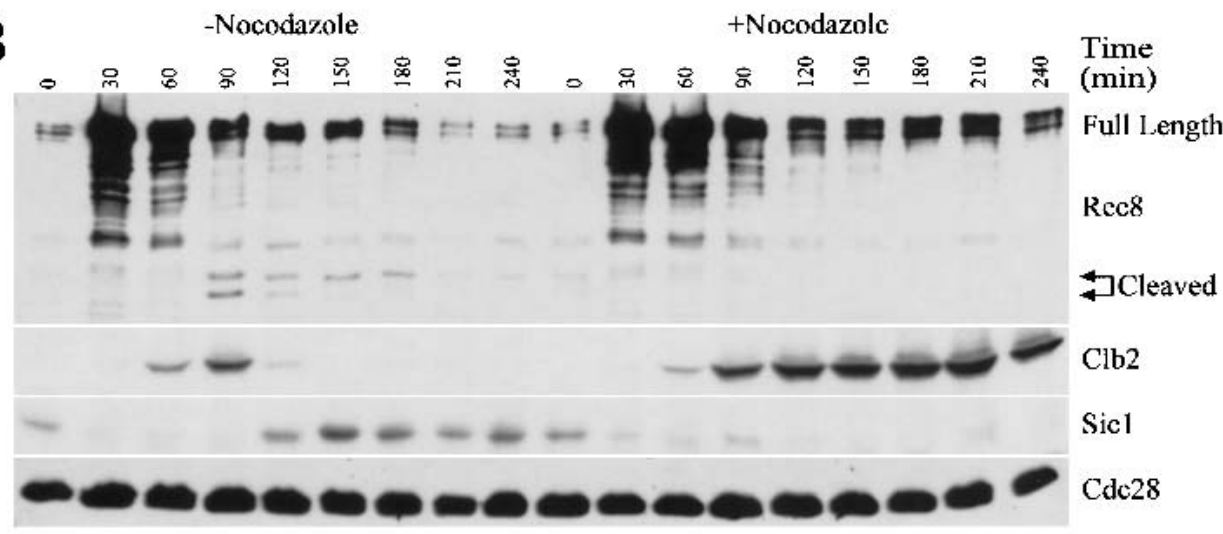

$\mathrm{C}$

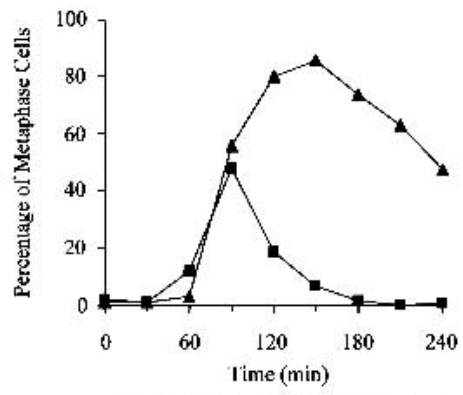

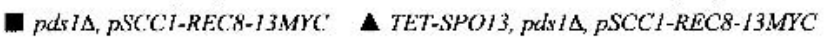

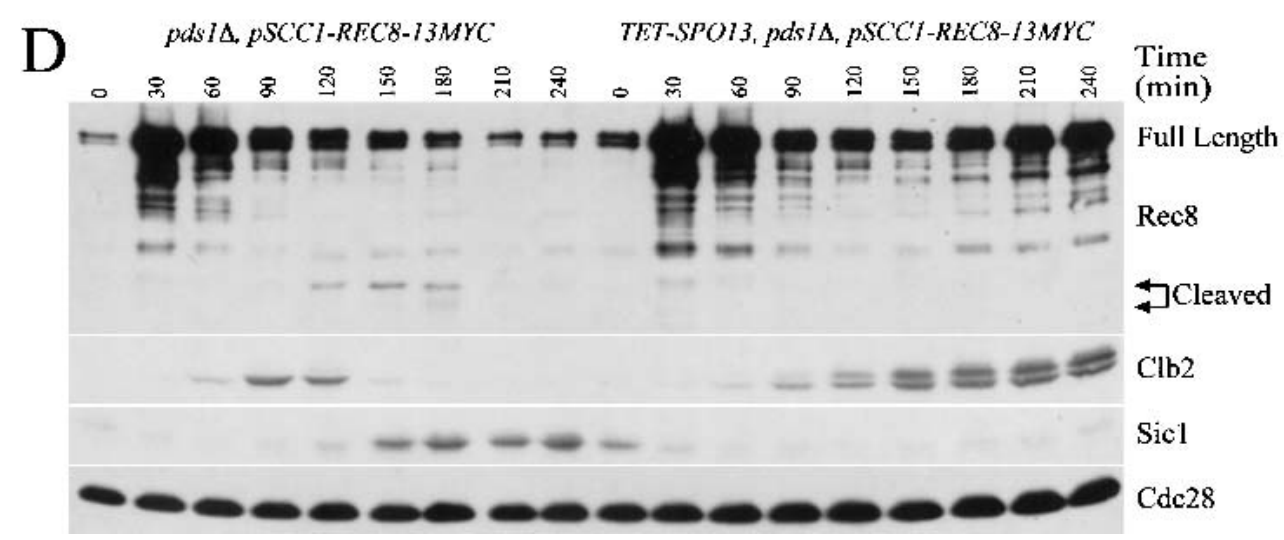

Figure 3. Spo13 inhibits cleavage of Rec8. $(A, B)$ pSCC1-REC8-13MYC (ద, A5468) cells were grown as described in Figure 1B except that after release from $\alpha$-factor arrest, the culture was split and $15 \mu \mathrm{g} / \mathrm{mL}$ nocodazole was added to one-half of the cells. After $110 \mathrm{~min}$, when cells had budded, $5 \mu \mathrm{g} / \mathrm{mL} \alpha$-factor was added to prevent entry into the second cell cycle. The percentage of cells with metaphase (A, left graph) or anaphase ( $A$, right graph) spindles and the total amount of Rec8-Myc, Clb2, and Sic1 were determined at the indicated

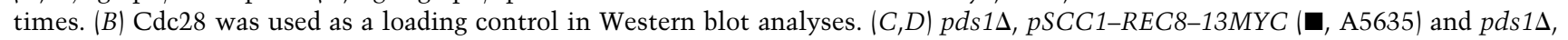
pSCC1-REC8-13MYC, TET-SPO13 ( $\mathbf{\Lambda}$, A5645) cells were grown as described in Figure 1B, and the percentage of cells with metaphase (C, left graph) or anaphase (C, right graph) spindles was determined at the indicated times. $(D)$ The total amount of Rec8-Myc, Clb2, and Sic1 was determined by Western blot analysis. Cdc28 was used as a loading control. 

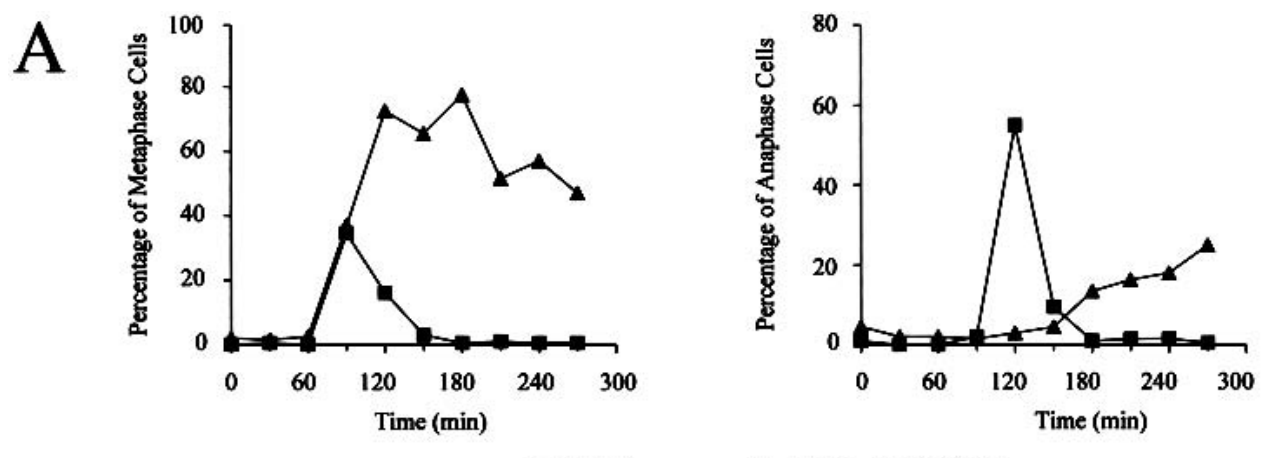

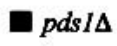

A pds1A, TET-SPOI3

B
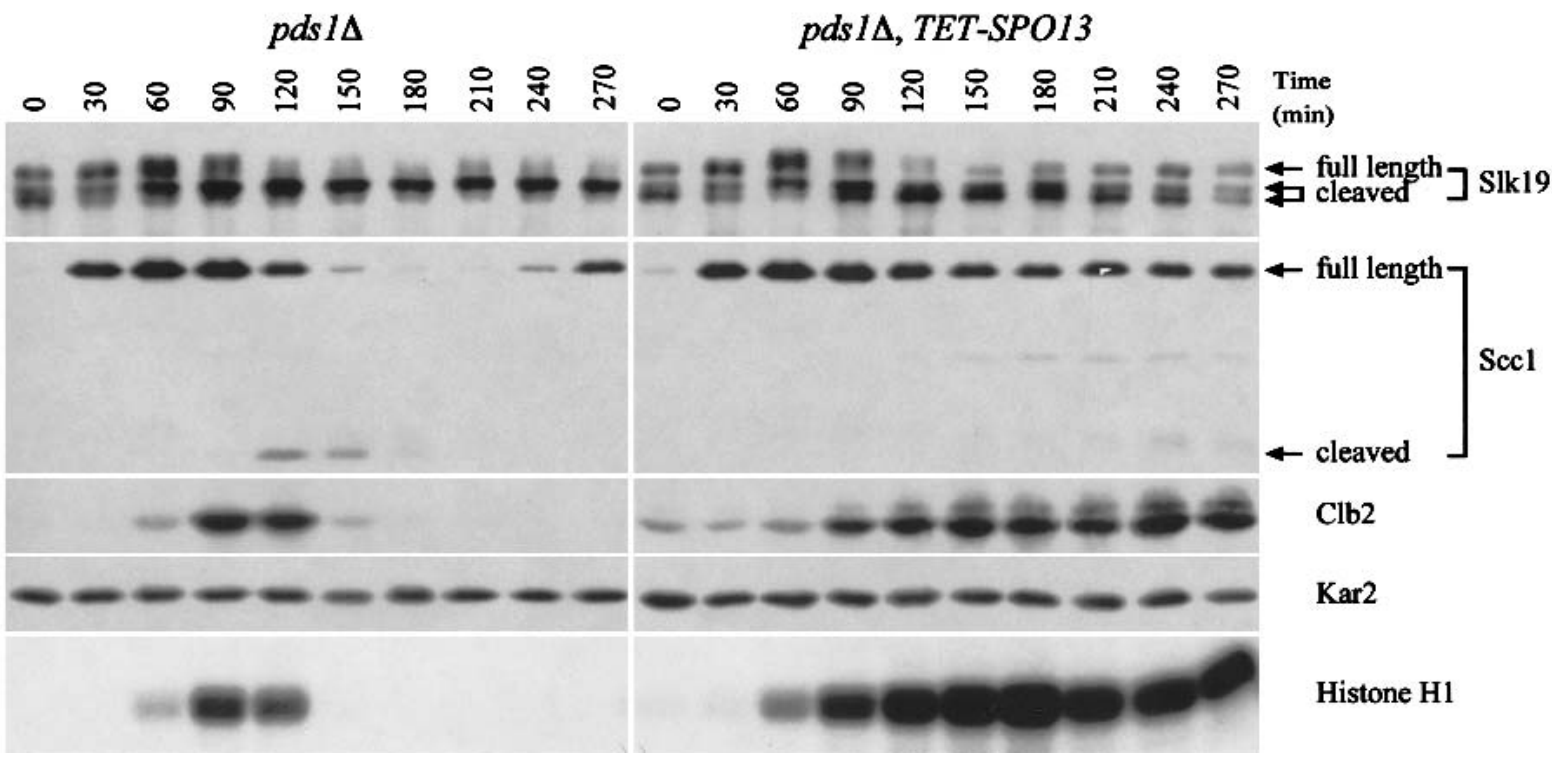

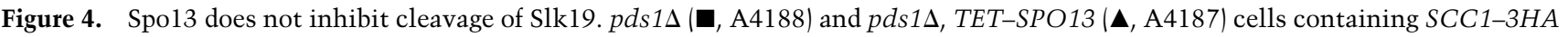
and SLK19-13MYC fusions were grown as described in Figure 1B to determine the percentage of cells with metaphase (A, left graph) or anaphase (A, right graph) spindles. (B) The total amount of Scc1-3HA, Slk19-13MYC, and Clb2 was determined by Western blot analysis. Clb2-associated kinase activity was determined using Histone $\mathrm{H} 1$ as a substrate. At the 0 time point, three forms of Slk19-13MYC are visible. The upper band is full-length Slk19, which becomes phosphorylated as cells progress through the cell cycle. The bottom band corresponds to the C-terminal cleavage product of Slk19; and the middle band is the phosphorylated form of the cleavage product.

stabilization, suggesting that the effects of SPO13 overexpression during mitosis to some extent recapitulate Spo13's function during meiosis. This observation, together with the finding that Rec8 is lost at centromeres during meiosis I in the absence of SPO13 (Klein et al. 1999|, suggests that Spo13 is an important factor in the control of cohesin cleavage in meiosis.

\section{SPO13 specifically inhibits Scc1/Mcd1 and Rec8 cleavage when overexpressed during mitosis}

Overexpression of Spo13 inhibited Scc1/Mcd1 and Rec8 but not Slk19 cleavage. The finding that Spo13 can prevent cleavage of a subset of separase substrates raises the interesting possibility that mechanisms exist that determine whether or not a substrate is cleaved by separase. Modifications such as phosphorylation on Espl itself
(Stemmann et al. 2001) could determine whether or not Esp1 could recognize a certain substrate. Alternatively, substrate modifications and/or accessibility could be the source of specificity. Phosphorylation of Scc1/Mcd1 by the polo-like kinase Cdc5 has been shown to greatly enhance Esp1's ability to cleave Scc1/Mcd1 (Alexandru et al. 2001). Perhaps Cdc5-dependent phosphorylation or other modifications are important to induce cleavage of some substrates but not others.

\section{How does SPO13 prevent Scc1/Mcd1 or Rec8 cleavage?}

As high levels of SPO13 inhibit Scc1/Mcd1 and Rec8 cleavage but not that of another Esp1 substrate, S1k19, it is clear that Spo13 does not generally inhibit Esp1 activity, but rather specifically prevents Scc1/Mcd1 or Rec8 from being cleaved. The mechanism whereby Spo13 pro- 
tects Scc1/Mcd1 or Rec8 from being cleaved, however, remains obscure. One possibility we considered was that Spo13 affects the function of Cdc5, a protein kinase that aids Esp1 in cleaving Scc1/Mcd1 (Alexandru et al. 2001). The finding that overexpression of CDC5 did not lead to Scc1/Mcd1 cleavage in cells with high levels of Spo13 whereas overexpression of ESP1 did (B. Lee, unpubl.) argues against this possibility. Another possibility is that Spo13 binds directly to the cohesin complex and occludes access of Esp1 to its substrate. This hypothesis would predict that Spo13 and components of the cohesin complex physically interact. We have not observed such a physical interaction (B. Lee, unpubl.). Additionally, Spo13 does not prevent Scc1-Tev268 from being cleaved by the TEV protease, suggesting that the TEV cleavage site and presumably the Esp 1 site that the TEV cleavage site replaces are not physically obscured by Spo13. Therefore, we believe that Spo13 does not itself prevent Esp1 from cleaving Scc1/Mcd1 or Rec8 but plays a regulatory role in this process.

Further support for the idea that Spo13 functions in a regulatory manner comes from the multiple phenotypes associated with SPO13 overexpression during the mitotic cell cycle. Spo13 not only inhibited cohesin cleavage but also prevented mitotic CDK inactivation. This inability to inactivate mitotic CDKs is not an indirect consequence of GAL-SPO13 or TET-SPO13 cells failing to separate sister chromatids. When Spo13's inhibitory effect on sister-chromatid separation was alleviated, either by using a temperature-sensitive mcd1-1 allele or by inducing Scc1/Mcd1 cleavage by the TEV protease, mitotic CDK inactivation was still delayed or prevented. The inhibition of mitotic CDK inactivation brought about by high levels of Spo13 was also not an indirect consequence of a delay in Pds1 degradation, as Spo13 also prevented mitotic CDK inactivation in cells lacking PDS1. How Spo13 regulates sister-chromatid cohesion and mitotic CDK activity is still unclear. It is, however, interesting to note that the protein phosphatase Cdc14 is required for mitotic $\mathrm{CDK}$ inactivation and for accurate meiotic chromosome segregation (Sharon and Simchen 1990; Visintin et al. 1998; Jaspersen et al. 1999). Perhaps Spo13 exerts its multiple functions through the regulation of Cdc14.

\section{Materials and methods}

All strains were derivatives of the yeast strain W303 (A2587). The TET-SPO13 construct was created by ligating a PCR fragment containing the SPO13 open reading frame (ORF) into pCM190 (Gari et al. 1997). Subsequently, the $2 \mu$ origin in pCM190 was deleted creating an integration plasmid that was then $3 \mathrm{x}$-integrated at the URA3 locus. The GAL-SPO13-TRP1 fusion was constructed by ligating the SPO13 ORF under the control of the GAL1-10 promoter fragment. The fusion was subsequently cloned into YIplac204 (Gietz and Sugino 1988) and integrated at the TRP1 locus. The SCC1-3HA, SLK19-13MYC, and REC8-13MYC fusions were constructed by a one-step PCR method (Longtine et al. 1998). To clone REC8 under the control of the SCC1 promoter (pSCC1-REC8-13MYC), the GAL1-10 promoter fragment in pFA6a-pGAL (Longtine et al. 1998) was replaced by a 1-kb SCC1-promoter PCR fragment. The resulting plasmid was used as a template in a one-step PCR-based method (Longtine et al. 1998) to replace the endogenous REC8 promoter in a REC8-13MYC strain with the SCC1 promoter. SCC1$18 M Y C$ and PDS1-HA constructs were described in Michaelis et al. (1997) and Cohen-Fix et al. (1996), respectively.

Growth conditions for individual experiments are described in the figure legends. Unless otherwise noted, cells were grown at $25^{\circ} \mathrm{C}$. Immunoblot analysis was performed as described in Cohen-Fix et al. (1996). To visualize Rec8 and its cleavage products, extracts were prepared by precipitating cells with trichloroacetic acid (TCA) and lysed as described in Moll et al. (1991). Clb2-associated kinase activity was assayed as described in Surana et al. (1993). Indirect in situ immunofluorescence methods and antibody concentrations are described in Visintin et al. (1999). Metaphase spindles were defined as mitotic spindles spanning an undivided nucleus. Anaphase spindles were defined as mitotic spindles spanning a divided nucleus.

\section{Acknowledgments}

We are grateful to Marion Shonn and Andrew Murray for communication of results prior to publication; Frank Uhlmann for many suggestions and reagents; Kim Nasmyth for yeast strains; and Mike Tyers and Mark Rose for antibodies. We thank Terry Orr-Weaver, Frank Solomon, and members of the Amon Lab for their critical reading of the manuscript. This research was supported by a National Institute of Health grant GM 62207 to A.A. B.L. is supported by a NSF pre-doctoral fellowship.

The publication costs of this article were defrayed in part by payment of page charges. This article must therefore be hereby marked "advertisement" in accordance with 18 USC section 1734 solely to indicate this fact.

\section{References}

Alexandru, G., Uhlmann, F., Mechtler, K., Poupart, M.A., and Nasmyth, K. 2001. Phosphorylation of the cohesin subunit Scc1 by Polo/Cdc5 kinase regulates sister chromatid separation in yeast. Cell 105: 459-472.

Buonomo, S.B., Clyne, R.K., Fuchs, J., Loidl, J., Uhlmann, F., and Nasmyth, K. 2000. Disjunction of homologous chromosomes in meiosis I depends on proteolytic cleavage of the meiotic cohesin Rec8 by separin. Cell 103: 387-398.

Cohen-Fix, O. 2001. The making and breaking of sister chromatid cohesion. Cell 106: 137-140.

Cohen-Fix, O., Peters, J.-M., Kirschner, M.W., and Koshland, D. 1996. Anaphase initiation in Saccharomyces cerevisiae is controlled by the APC-dependent degradation of the anaphase inhibitor Pds1p. Genes \& Dev. 10: 3081-3093.

Gardner, R.D. and Burke, D.J. 2000. The spindle checkpoint: Two transitions, two pathways. Trends Cell Biol. 10: 154158.

Gari, E., Piedrafita, L., Aldea, M., and Herrero, E. 1997. A set of vectors with a tetracycline-regulatable promoter system for modulated gene expression in Saccharomyces cerevisiae. Yeast 13: 837-848.

Gietz, R.D. and Sugino, A. 1988. New yeast-Escherichia coli shuttle vectors constructed with in vitro mutagenized yeast genes lacking six-base pair restriction sites. Gene 74: 527534.

Guacci, V., Koshland, D., and Strunnikov, A. 1997. A direct link between sister chromatid cohesion and chromosome condensation revealed through the analysis of MCD1 in S. cerevisiae. Cell 91: 47-57. 
Hugerat, Y. and Simchen, G. 1993. Mixed segregation and recombination of chromosomes and YACs during single-division meiosis in spo13 strains of Saccharomyces cerevisiae. Genetics 135: 297-308.

Jaspersen, S.L., Charles, J.F., and Morgan, D.O. 1999. Inhibitory phosphorylation of the APC regulator Hct1 is controlled by the kinase Cdc28 and the phosphatase Cdc14. Curr. Biol. 9: 227-236.

Klapholz, S. and Esposito, R.E. 1980a. Isolation of SPO12-1 and SPO13-1 from a natural variant of yeast that undergoes a single meiotic division. Genetics 96: 567-588.

- 1980b. Recombination and chromosome segregation during the single division meiosis in SPO12-1 and SPO13-1 diploids. Genetics 96: 589-611.

Klein, F., Mahr, P., Galova, M., Buonomo, S.B., Michaelis, C., Nairz, K., and Nasmyth, K. 1999. A central role for cohesins in sister chromatid cohesion, formation of axial elements, and recombination during yeast meiosis. Cell 98: 91-103.

Lee, J.Y. and Orr-Weaver, T.L. 2001. The molecular basis of sister-chromatid cohesion. Annu. Rev. Cell Dev. Biol. 17: 753-777.

Longtine, M.S., McKenzie III, A., Demarini, D.J., Shah, N.G., Wach, A., Brachat, A., Philippsen, P., and Pringle, J.R. 1998. Additional modules for versatile and economical PCR-based gene deletion and modification in Saccharomyces cerevisiae. Yeast 14: 953-961.

McCarroll, R.M. and Esposito, R.E. 1994. SPO13 negatively regulates the progression of mitotic and meiotic nuclear division in Saccharomyces cerevisiae. Genetics 138: 47-60.

Melo, J. and Toczyski, D. 2002. A unified view of the DNAdamage checkpoint. Curr. Opin. Cell Biol. 14: 237-245.

Michaelis, C., Ciosk, R., and Nasmyth, K. 1997. Cohesins: Chromosomal proteins that prevent premature separation of sister chromatids. Cell 91: 35-45.

Miyazaki, W.Y. and Orr-Weaver, T.L. 1994. Sister-chromatid cohesion in mitosis and meiosis. Annu. Rev. Genet. 28: 167187.

Moll, T., Tebb, G., Surana, U., Robitsch, H., and Nasmyth, K. 1991. The role of phosphorylation and the CDC28 protein kinase in cell cycle-regulated nuclear import of the $S$. cerevisiae transcription factor SWI5. Cell 66: 743-758.

Nasmyth, K., Peters, J.-M., and Uhlmann, F. 2000. Splitting the chromosome: Cutting the ties that bind sister chromatids. Science 288: 1379-1385.

Peters, J.-M. 1998. SCF and APC: The Yin and Yang of cell cycle regulated proteolysis. Curr. Opin. Cell Biol. 10: 759-768.

Salah, S.M. and Nasmyth, K. 2000. Destruction of the securin Pds1p occurs at the onset of anaphase during both meiotic divisions in yeast. Chromosoma 109: 27-34.

Severin, F., Hyman, A.A., and Piatti, S. 2001. Correct spindle elongation at the metaphase/anaphase transition is an APCdependent event in budding yeast. J. Cell Biol. 155: 711-718.

Sharon, G. and Simchen, G. 1990. Mixed segregation of chromosomes during single-division meiosis of Saccharomyces cerevisiae. Genetics 125: 475-485.

Stemmann, O., Zou, H., Gerber, S.A., Gygi, S.P., and Kirschner, M.W. 2001. Dual inhibition of sister chromatid separation at metaphase. Cell 107: 715-726.

Sullivan, M., Lehane, C., and Uhlmann, F. 2001. Orchestrating anaphase and mitotic exit: Separase cleavage and localization of Slk19. Nat. Cell Biol. 3: 771-777.

Surana, U., Amon, A., Dowzer, C., Mcgrew, J., Byers, B., and Nasmyth, K. 1993. Destruction of the CDC28/CLB kinase is not required for metaphase/anaphase transition in yeast. EMBO J. 12: 1969-1978.

Tang, T.T., Bickel, S.E., Young, L.M., and Orr-Weaver, T.L.
1998. Maintenance of sister-chromatid cohesion at the centromere by the Drosophila MEI-S332 protein. Genes \& Dev. 12: 3843-3856.

Toth, A., Rabitsch, K.P., Galova, M., Schleiffer, A., Buonomo, S.B., and Nasmyth, K. 2000. Functional genomics identifies monopolin: A kinetochore protein required for segregation of homologs during meiosis I. Cell 103: 1155-1168.

Uhlmann, F. 2001. Chromosome cohesion and segregation in mitosis and meiosis. Curr. Opin. Cell Biol. 13: 754-761.

Uhlmann, F. and Nasmyth, K. 1998. Cohesion between sister chromatids must be established during DNA replication. Curr. Biol. 8: 1095-1101.

Uhlmann, F., Lottspeich, F., and Nasmyth, K. 1999. Sister-chromatid separation at anaphase onset is promoted by cleavage of the cohesin subunit Scc1. Nature 400: 37-42.

Uhlmann, F., Wernic, D., Poupart, M.A., Koonin, E.V., and Nasmyth, K. 2000. Cleavage of cohesin by the CD clan protease separin triggers anaphase in yeast. Cell 103: 375-386.

Visintin, R., Craig, K., Hwang, E.S., Prinz, S., Tyers, M., and Amon, A. 1998. The phosphatase Cdc14 triggers mitotic exit by reversal of CDK-dependent phosphorylation. Mol. Cell 2: 709-718.

Visintin, R., Hwang, E.S., and Amon, A. 1999. Cfil prevents premature exit from mitosis by anchoring Cdc14 phosphatase in the nucleolus. Nature 398: 818-823. 


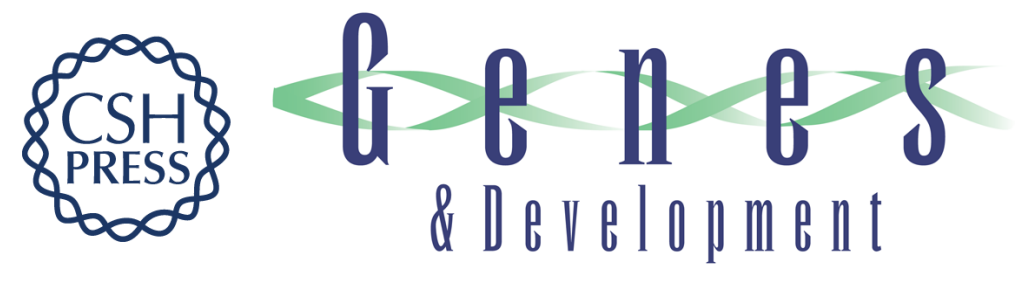

\section{Spo13 regulates cohesin cleavage}

Brian H. Lee, Angelika Amon and Susanne Prinz

Genes Dev. 2002, 16:

Access the most recent version at doi:10.1101/gad.989302

References This article cites 36 articles, 9 of which can be accessed free at: http://genesdev.cshlp.org/content/16/13/1672.full.html\#ref-list-1

License

Email Alerting Receive free email alerts when new articles cite this article - sign up in the box at the top Service right corner of the article or click here.

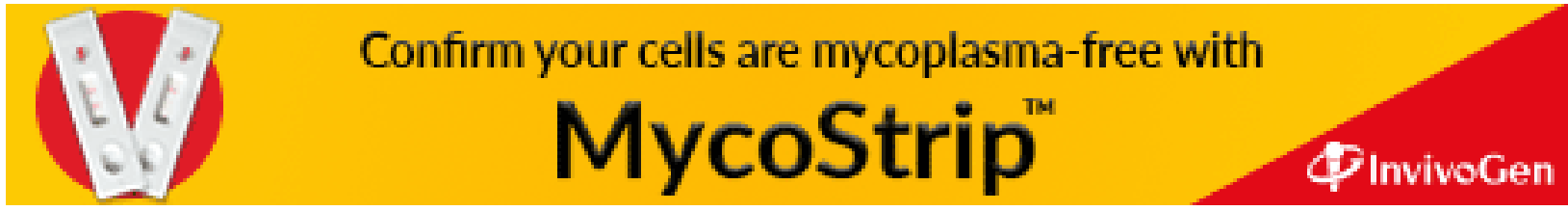

